[March,

from the four inflexional lines that meet, e. g., $\overline{A B}$ and $\bar{A} \bar{C}$, in inflexional points other than $A$.

The nonsingular plane cubic is one of an indefinitely great series of "elliptic normal curves" in spaces of three dimensions, four dimensions, etc., respectively. On each of these there exists a configuration analogous to that of the inflexion-system of the cubic. All such are mere matters of course when the points of the curve are represented by values of an elliptic integral of the first sort. But they are no less easy of discovery by the immediate extension of the foregoing method; and the closed chains of four tetrahedra, five pentahedra, etc. appear to be novel and interesting objects for the geometric imagination. Further, by the application of elliptic parameters to these objects an extensive theory may be evolved, peculiar and not devoid of profit.

NORTH WESTERN UNIVERSITY, December, 1897.

\title{
ON THE INTERSECTIONS OF PLANE CURVES.
}

BY PROFESSOR CHARLOTTE ANGAS SCOTT.

Linea Ordinis $(n)$ occurrere potest aliæ ejusdem Ordinis in punctis $n^{2}$. Proinde duæ Lineæ Ordinis $(n)$ per eadem puncta $n^{2}$ transire nonnunquam possunt; adeoque puncta data quorum numerus est $\frac{1}{2}\left(n^{2}+3 n\right)$ non sufficiunt ad lineam Ordinis $(n)$ ita determinandam ut unica sit curva quæ per ea data puncta duci possit: Cum vero coefficientes in æequatione generali ad Lineam Ordinis $(n)$ sint $\frac{1}{2}\left(n^{2}+3 n\right)$, patet si plura dentur puncta, Lineam Ordinis $(n)$ per ea forsan duci non posse et Problema reddi posse impossibile. Sic novem puncta non adeo plene determinant Lineam Ordinis tertii ac quinque Lineam Ordinis secundi, decem tamen ad Lineam tertii Ordinis determinandam nimia sunt.

Maclaurin, Geometria Organica, 1720 ; Sect. V, Lemma III, Corol : II ; p. 137.

Ensuite je ferai voir le défaut, qui se trouve dans ces conséquences, qui consiste dans une fort subtile précipitation du raisonnement, laquelle n'etant pas si facile à découvrir, nous doit rendre extrémement circonspects, principalement dans les autres sciences, afin que nous ne nous laissions pas séduire par de semblables contradictions apparentes. Car, 
si dans la Géométrie nous sommes assujettis à des difficultés si remarquables, où il est pourtant permis de ramener toutes les idées presque au plus haut degré de justesse, combien plus pourrons-nous être embarrassés dans les autres sciences, où il n'est pas possible de parvenir à des idées assez précises, et où il est infiniment plus difficile de se garantir de pareilles fautes dans le raisonnement?

EuLER, Sur une contradiction apparente dans la doctrine des lignes courbes, 1748-50, p. 221.

Cette contradiction se léve par la Remarque qui termine le $\$ 38$. C'est qu'encore qu'on ait autant d'équations qu'il en faut, généralement parlant, pour déterminer tous les coëfficients de l'équation prise pour réprésenter la Courbe qui doit passer par un certain nombre de points donnés, il peut pourtant arriver que ces coëfficients restent indéterminés. Alors l'équation prise reste indéterminée et réprésente une infinité de Courbes du même Ordre. *** Ce qui est un véritable paradoxe.

Cramer, Introduction à l'Analyse des Lignes Courbes algébriques, 1750 ; Ch. III, § 48, p. 79.

In mathematischen Dingen kann nicht lange etwas ein Paradox bleiben, eben so wenig als ein Kunstgriff lange als solcher sich behaupten kann; das Paradox verschwindet, wenn verdeckte Mittelglieder der Verkettung mathematischer Sätze hervortreten und der Kunstgriff verliert sich in einer neuen durchgreifenden Behandlungsweise.

Plücker, Theorie der Algebraischen Curven, 1839 ; p. 12.

Es dauerte einige Zeit, bis dieses so genannte Paradoxon seiner wahren Bedeutung nach, nämlich als Quelle von Sätzen, erkannt wurde.

Clebsch, Zum Gedächtniss an Julius Plücker, 1871.

The theory of the intersections of curves has probably led its investigators into more errors than any other modern theory. Even the history of the central question, the socalled Cramer paradox, is usually given incorrectly, with the omission of all reference to Maclaurin. This is the more surprising, inasmuch as Cramer himself ascribes to Maclaurin the theorem that the $n^{2}$ intersections of two curves of order $n$ impose only a certain number of conditions, and gives the exact reference. Plücker was familiar with the passage in Cramer; nevertheless he overlooks Maclaurin, though he was sufficiently interested historically in the Cramer paradox to give also the reference to Euler. 
He brings his account up to date with a mention of his own "Théorèmes généraux concernant les équations d'un degré quelconque entre un nombre quelconque d'inconnues,", C'relle's Journal, vol. 16, pp. 47-57, 1837, and Jacobi's "De relationibus, quæ locum habere debent inter puncta intersectionis duarum curvarum vel trium superficierum algebraicarum dati ordinis, simul cum enodatione paradoxi algebraici," Crelle's Journal, vol. 15, pp. 285-308, 1836, stating that both memoirs were in the editor's hands at the same time. Clebsch, in his "Gedächtnissrede," though speaking from minute first-hand knowledge of Cramer, makes no mention of Maclaurin ; in Clebsch-Lindemann's Vorlesungen über Geometrie, 1876, p. 426, and in Salmon's Higher Plane Curves, $3 \mathrm{~d}$ ed., 1879 , p. 22 , the account is substantially that of Plücker, and thus Maclaurin is ignored here, as also in Loria's Il passato ed il presente delle principali teorie geometriche, $2 \mathrm{~d}$ ed., 1896. Brill and Noether, however, supply the reference in their Bericht, "Die Entwicklung der Theorie der algebraischen Functionen in älterer und neuerer Zeit,"' 1894, Jahresberichte der Deutschen Mathematiker-Vereinigung, vol. 3, p. 130 ; and as it is given also in the analysis of the Geometria Organica in Cantor's Vorlesungen über Geschichte der Mathematik, vol 3 (Part 2) 1896, pp. 426, 427, Maclaurin will probably obtain due credit on this score in future.

Maclaurin's precise understanding is that the $\frac{1}{2} n(n+3)$ points assigned for the determination of a curve of order $n$, if included in the $n^{2}$ points given by the intersections of two curves of order $n$, will fail to determine the curve uniquely; he makes no attempt to explain it. Cramer goes further than this; he gives the explanation, and says that an infinity of curves can be found satisfying the given conditions, a result arrived at by algebraic considerations. $\mathrm{He}$ does not formulate the conclusion that a certain number of the nine or sixteen points (he speaks principally of cubic and quartic curves) are a necessary consequence of the remainder, though Clebsch, es roneously according to Brill and Noether, assigns this view of the theorem to him. The actual wording does not disprove his knowledge of this, nor does it necessarily imply it; but it is plain that the fact, even if he noticed it, did not strike him as the important one in the theorem. Naturally he does not recognize the linear nature of the system of curves that can be found satisfying the given conditions, nor does he realize that only one indeterminate quantity is involved, as may be clearly seen on p. 60 ; this particular step seems to 
have been taken by Lamé, who expressed the system of curves in the form $m E+m^{\prime} E^{\prime}=0$, Examen des différentes méthodes employées pour résoudre les problèmes de géométrie, 1818, p. 28. (See also the Brill-Noether Bericht, pp. 289 et. sq., and Plücker's Collected Papers, vol. 1, p. 597 , Schoenflies, for other historical references.)

Euler's resolution of the paradox, though published in the same year as that of Cramer, was communicated to the Berlin Academy two years earlier. It agrees with that of Cramer, and goes just as far, or even a little further, if we give the most liberal interpretation to a sentence on p. 232 . Euler is decidedly lavish of explanation, with his Propositions I, II, Difficulties I, II, III, and Reflexions I, II, III, IV, all preliminary to the illustrations by means of which he explains his point. The memoir bears the title "Sur une contradiction apparente dans la doctrine des lignes courbes," Mémoires de l'Academie Royale des Sciences et Belles Lettres, Berlin (1748), vol. 4, 1750, pp. 219-233.*

The allied theorem that if of the $n^{2}$ intersections of two curves of order $n, n p$ lie on a curve of order $p$, the remainder lie on a curve of order $n-p$, was given by Gergonne, $A n$ nales de Mathématiques, vol. 17, p. 220, 1827. In vol. 19 of the same periodical there is a short memoir by Plücker, " Recherches sur les courbes algébriques de tous les degrés," in which the algebraic view of the Cramer paradox, namely, that from a given system of equations others of the same type are derivable, is applied to the theory of conjugate points with respect to a conic. A more important contribution to the theory is contained in the Analytisch-geometrische Entwickelungen, vol. 1, 1828, p. 228 (foot note), where one curve through the points is regarded as fixed. Clebsch speaks of this as an indispensable preliminary to any advance in the theory ; Brill and Noether, in reproducing this judgment, p. 290, modify it somewhat, simply emphasizing the importance of the convention for the theory of functions, with a tacit recognition of the fact that the significance of the paradox is not confined to this.

The investigations associated with this "Quelle von

* This memoir is immediately followed by the "Démonstration sur le nombre des points ou deux lignes des ordres quelconques peuvent se couper," pp. 234-248, in which the number of intersections, $m n$, arrived at by induction by Maclaurin, is proved by the process of elimination by symmetric functions. This is the only one of the two memoirs explicitly mentioned in the Brill-Noether Bericht, pp. 140, 143 ; the first is, however, the one referred to, by content not by title, on pp. 138, 289, though the cross-reference to p. 138 on p. 143 would lead one to suppose that the second memoir is intended. 
Sätzen " fall into three divisions, which for convenience will be referred to as German, Italian, and English. The first is virtually the Brill-Noether work, originating with the memoir " Ueber die algebraischen Functionen und ihre Anwendung in der Geometrie," Mathematische Annalen, vol. 7, pp. 269-310, 1873, in which the consequences, immediate and remote, are considered in the light of the theory of functions. The one curve, fixed by Plücker, is primarily the locus in quô ; on this certain groups of points are selected by purely analytical characteristics which they have in common ; the subject of investigation is the system of groups of points so determined, and it is by a rather grudging concession that these are spoken of as cut out by adjoint curves ; notwithstanding the important geometrical applications of the theory, geometry is little more than an accessory after the fact.

In the Italian work, on the contrary, as exhibited in the series of memoirs by Caporali, Jung, Bertini,* Guccia, Segre, Castelnuovo, and others, the geometrical view is paramount. The subject of investigation is the linear system of curves of any assigned order, determined by given fixed points; in intimate connection with this there are certain derived systems. While the groups of points cut out by the system on any one curve-fixed only for the moment in this class of investigations-do in fact present themselves, yet they are of quite secondary interest.

It is not easy to give a concise description of the investigations of the third division. They concern themselves hardly at all with a system of groups of points on one curve, hardly at all with a system of curves through one group. The locus in quô is the plane; the subject of investigation is the group of points, the complete or partial intersection of two curves of the same or different orders, regarded as a means of imposing conditions on another curve of any order. In speaking of this as the English theory, I do not wish to imply any geographical restriction, but simply to emphasize the fact that of the three classes of investigation, this is the one chiefly recognized in English works. (See Salmon's Higher Plane Curves, $\$ \$ 28-34$.) Hitherto it has been hardly more than a collection of detached theorems, of the type of Cayley's well-known intersection theorem, growing directly out of the simpler theorems of Maclaurin on the

* The greater part of one of Bertini's memoirs, "La geometria delle serie lineari sopra una curva piana secondo il metodo algebrico," Annali di Matematica, Ser. II., vol. 22, pp. 1-40, 1894, is devoted to an exposition of the Brill-Noether theory, and hence belongs to the first division. 
intersections of curves, these theorems, in virtue of the simplicity of their derivation, proving immediately fruitful in applications to geometry. $*$ Inasmuch as the groups of points that occur are connected by a curve through two or more, there are relations to the German theory ; inasmuch as the two or more curves that are passed through a group may be taken of the same order, so belonging to a linear system of curves, there is something in common with the Italian theory ; and coincidences of results are to be expected. That the various detached theorems are of importance, not only in their direct geometrical application, but also as products of a process of value, has, I think, been abundantly proved by the latest memoir in this line, "Point-Groups in relation to Curves," by Mr. F. S. Macaulay, in the Proceedings of the London Mathematical Society, 1895, vol. 26 , pp. $495-544$.

Cayley's theorem, that a curve of order $n$ through all but $\frac{1}{2}(l+m-n-1)(l+m-n-2)$ of the points common to two curves of orders $l$ and $m$ will necessarily pass through the remainder if $n$ be not less than $l$ or $m$ but less than their sum (Collected Papers, vol. 1, no. 5), was given in a short paper in the Cambridge Mathematical Journal, vol. 3, 1843. Clebsch in 1871 ventured the opinion that with this the algebraic side of the investigation of the Cramer paradox can be regarded as closed. But this was disproved ten years later, when Bacharach, in his inaugural dissertation, "Ueber Schnittpunktsysteme algebraischer Curven" (Erlangen), incorporated in the memoir "Ueber den Cayley'schen Schnittpunktsatz,' Mathematische Annalen, vol. 26, pp. 275299,1886 , showed that a specialization in the position of the $\delta$ points $\left[\delta=\frac{1}{2}(l+m-n-1)(l+m-n-2)\right]$, would affect the theorem. To the recognition of this possible specialization belongs the credit of much of the later geometrical work. Bacharach's form of the theorem is :-a curve of order $n$ through $l m-\delta$ of the points will of necessity pass through the remaining $\delta$, unless these lie on a curve of order $l+m-n-3$. This modification was discussed and adopted by Cayley in the memoir "On the Intersections of Curves" (Collected Papers, vol. 12, no. 868), Mathematische Annalen, vol. 30, 1887 ; a more detailed proof

\footnotetext{
* See for example Cremona, Introduzione ad una teoria geometrica delle curve piane, 1862 , p. 39 ; Olivier, in Crelle, vols. 70, 71, 1869, 1870 ; Study, Math. Ann., vol. 36, 1890. In this connection it is perhaps not superfluous to call attention to Bacharach's remark, Math. Ann., vol. 26, p. 295, that the professed extensions of Olivier's theorems given on pp. 762-764 of Clebsch-Lindemann are not legitimate; it may be added that the whole of Sect. V. of Chap. 6, pp. 753-764, needs correction.
} 
was given by Zeuthen, "Sur la détermination d'une courbe algébrique par des points donnés," Mathematische Annalen, vol. 31,1888 . It is necessary, in considering the series of theorems of which this is the type, to keep the two parts distinct (see Brill-Noether Bericht, p. 290). The expression for the postulation, to use Cayley's term, of the $l m$ points, namely,

$$
D=l m-\delta=l m-\frac{1}{2}(l+m-n-1)(l+m-n-2),
$$

is true without exception ; but it is not true without exception that the $\delta$ points necessarily follow from the $D$ points. It may be the case that the $l m$ points consist of $D_{1},<D$, with an induced number $\delta_{1}$, and other points. Thus for example the sixteen intersections of two quartics, one of which consists of a cubic and a straight line, are not such that any three follow from the thirteen ; from eleven of the intersections of the cubic and quartic the twelfth follows; from these twelve with two (arbitrary) points, the fifteesth and sixteenth follow.

Mr. Macaulay introduces the convenient term $n$-ic excess for the excess of the number of points in any one group over the number of independent conditions that these points impose on $C_{n}$, a curve of order $n$. Thus if $N$ be the number of points, $D$ their postulation for $n$-ics, the $n$-ic excess, $r_{n}$, $=N-D$. In Castelnuovo's "Ricerche generali sopra i sistemi lineari di curve piane," Mem. della R. Acc. d. Sc. di Torino, 1891, Ser. II., vol. 42 , the analogous term sovrabbondanza is used with reference to the system of curves of any order determined by the points ; the system is said to be sovrabbondante; if there is no excess, the system is regolare. By the $n$-ic defect of the points, $q_{n}$, is to be understood the number of degrees of freedom of a curve of order $n$ through the points ; hence

$$
N-r_{n}+q_{n}=\frac{1}{2} n(n+3) .
$$

The statement of the first part of the Cayley theorem is then that if $n$ be not less than $l$ or $m$ the $n$-ic excess of $I(l, m) *$ is

$$
\left[\frac{1}{2}(l+m-n-1)(l+m-n-2)\right],
$$

where the square brackets indicate that the product so given presents itself only when the individual factors are positive. Thus if $n \geq l+m-2$, the $n$-ic excess is zero. The term

* I use this symbol to denote the eomplete intersection of two curves of orders $1, m$. 
multiplicity represents the Mannigfaltigkeit of the German work, that is, the index $q$ in the usual symbol $g_{Q}^{q}$; it is shown that the multiplicity of the system of groups cut out on a base-curve of order $m$ by curves of order $n$ through a group $N$ on the base-curve is

$$
q_{n}-\left[\frac{1}{2}(n-m+1)(n-m+2)\right] .
$$

In the course of the work the interesting fact is emphasized that if a group of points has an excess for a curve of any one order it has in general an excess for curves of other orders ; and that the $n$-ic excess for a given group diminishes as $n$ increases. The German and Italian investigations have not required the systematic consideration of curves of all orders through one group of points, so apparently the use that can be made of this fact has not been realized. It is shown that given the number of points, and the successive values of $r_{n}$, which together form the characterization of the group, its construction can be found. Such a group, one which owing to some interconnection of the points has an excess for at least one curve, is said to be of special form, this particular phrase being used because the simpler term special* is already appropriated in the German and Italian investigations.

* In the footnote to p. $499 \mathrm{Mr}$. Macaulay gives only the older use of the word special ; this was originally applied to groups $G_{Q}^{q}$ or systems $g_{Q}^{q}$ in which $Q-q<p-1$; see Brill and Noether, Math. Ann., vol. 7, p. 280; Clebsch-Lindemann, pp. 686, 699 ; Brill-Noether Bericht, p. 338. The later and more convenient usage, recognizing the more important ground of distinction in invariantic properties, makes special apply when

$$
Q-q \leq p-1,
$$

with some additional epithet for the restricted case $Q-q<p-1$; see Fiedler's translation of Salmon's Higher Plane Curves, 2d ed., 1882, p. 418 ; Castelnuovo, "Ricerche di geometria sulle curve algebriche," Atti della $R$. Acc. d. Sc. di Torino, 1889, vol. 24, \& 23 ; also "Ricerche generali," Cap. II., \& 18 ; Bertini, "La geometria delle serie lineari," \& 5, 21; Brill-Noether Bericht, pp. 359, 542 .

To a group of points which imposes fewer conditions on a curve of assigned order than would be supposed from the number of the points, Cayley's "special system," Collected Papers, vol. 7, p. 254, the term ausgezeichnete is applied (Brill-Noether Bericht, p. 543), the epithet having reference to a particular derivation of such a group; thus $\mathrm{Mr}$. Macaulay's point-group of special form is ausgezeichnete. Such special groups as are also ausgezeichnete, that is, groups for which $Q-q<p-1$, are spoken of as eigentlich special (Fiedler, p. 418, Brill-Noether Bericht, p. 547). I am not aware that any English equivalent has been suggested for eigentlich special, but one will surely be needed. Seeing that the term is applied to a class of cases, $Q-q<p-1$, included in the more extensive class special, $Q-q \leq p-1$, intraspecial appears suitable. 
If a group contains a point-group of special form, with other points that have no connection with this, it is said to be redundant; if while containing only a part of a pointgroup of special form, it contains so much as to oblige a curve to pass through the rest, it is incomplete; if it is neither redundant nor incomplete, it is complete. On this the criticism may be made that the completeness, redundancy, or incompleteness really depends on the order of the curve, instead of being, as the wording would imply, an intrinsic property of the point-group. Thus for example the 30 intersections of two curves of orders 5 and 6 impose $24,27,29$ conditions on curves of orders $6,7,8$; taking 28 of these 30 points, we have a group which is incomplete with reference to curves of orders 6 and 7 , but not with reference to curves of order 8. Moreover, a group may be at one and the same time both redundant and incomplete for any one curve, as for example the group of 14 points composed of the 12 intersections of a cubic and a quartic and 2 arbitrary points. The 12 intersections are a point-group of special form, which by the addition of the 2 arbitrary points is made redundant; but any quartic through the 14 will pass through other 2 fixed points, not derivable from the 2 alone; the group is therefore incomplete. This criticism, however, does not affect the general argument.

The process of proof is in general that of Zeuthen (1. c.). This is applied in the first few sections to establish the known theorems :

I. the $l$.ic excess of $I(l, m)=\frac{1}{2}(m-1)(m-2)$, where $l \geq m-2$;

II. any curve of order $n$ through $I(l, m)$ is of the form $C_{l} S_{n-l}+C_{m} S_{n-m}=0$, (Noether's fundamental theorem);

III. the theorem of Residuation ;

IV. the necessary and sufficient condition that a pointgroup on a $C_{m}$ lie on a $C_{m-3}$ is that the multiplicity be $\geq N-\frac{1}{2} m(m-3)$ with the corresponding result, equivalent to $Q-q \leq p-1$, for curves with multiple points.

In $\$ 18$ the Riemann-Roch theorem is given in the form:the multiplicity of any point-group on $C_{m}$ is equal to its $(m-3)$-ic excess. The Brill-Noether form of the theorem is expressed by a reciprocal relation between the defects and excesses, namely, if a point-group $N$ lie on a curve of order $m-3$, and have then a residual $N^{\prime}$, the $(m-3)$-ic defect and excess of $N$ are respectively equal to the ( $m-3)$-ic excess and defect of $N^{\prime}$; and the difference of the numbers $N, N^{\prime}$ is equal to twice the difference of the multiplicities. 
Among other results in $\$ 19$ there are two of particular importance for the following sections; they are obtained with the help of the Riemann-Roch theorem. ( $\$ 19$, iii.) If $N+N^{\prime}=I(m, n)$, and the $n$-ic excess of $N$ be not zero, then $N^{\prime}$ lies on a $C_{m-3}$ which does not pass through $N$; and if the $n$-ic excess of $N$ be zero, $N^{\prime}$ does not lie on a $C_{m-3}$; unless $n \leq m-3$, in which case any $C_{m-3}$ through $N^{\prime}$ necessarily passes through $N$. This is connected with the Bacharach version of the Cayley theorem. Writing $m=n$ it becomes the known theorem, stated in its most convenient form by Castelnuovo (Ricerche generali):-a system is regular or sovrabbondante according as the characteristic series is non-special or special. $\$ 19$, iv, the generalized form of no. iii, appears to be a new result, or, at any rate, if not absolutely new, it is shown in a new and more significant light. If $N+N^{\prime}=I(l, m)$, the lowest order of a curve that passes through $N^{\prime}$ without passing through $N$ is known when we know the highest order, $n$, of a curve through $N$ for which $N$ has an excess ; namely, it is $l+m-n-3$. Obviously curves of all higher orders can be passed through $N^{\prime}$ without passing through $N$, and it has been seen that for all lower values of $n$ the group $N$ will have an excess; hence what the theorem really shows is that according as $r_{n}>0$ or $=0$, a curve of order $l+m-n-3$ can or cannot be passed through $N^{\prime}$ without passing through $N$, and conversely.

In theorem V, $\$ 20$, the $(l+m-n-3)$-ic excess and defect of $N^{\prime}$ are expressed in terms of the $n$-ic defect and excess of $N$ in two equations, either of which is deducible from the other ; these are

$$
\begin{aligned}
r^{\prime}{ }_{l+m-n-3}=q_{n}+1 & -\left[\frac{1}{2}(n-l+1)(n-l+2)\right] \\
& -\left[\frac{1}{2}(n-m+1)(n-m+2)\right], \\
q^{\prime}{ }_{l+m-n-3}=r_{n}-1+ & {\left[\frac{1}{2}(l-n-1)(l-n-2)\right] } \\
+ & {\left[\frac{1}{2}(m-n-1)(m-n-2)\right] . }
\end{aligned}
$$

Writing $n^{\prime}$ for $l+m-n-3$, so that $n+n^{\prime}=l+m-3$, these relations become

$$
\begin{aligned}
r_{n^{\prime}}=q_{n}+1- & {\left[\frac{1}{2}(n-l+1)(n-l+2)\right] } \\
- & {\left[\frac{1}{2}(n-m+1)(n-m+2)\right], } \\
r_{n}=q_{n^{\prime}}+1- & {\left[\frac{1}{2}\left(n^{\prime}-l+1\right)\left(n^{\prime}-l+2\right)\right] } \\
- & {\left[\frac{1}{2}\left(n^{\prime}-m+1\right)\left(n^{\prime}-m+2\right)\right] . }
\end{aligned}
$$

When $l=n=n^{\prime}=m-3$, these reduce to the reciprocal relations already proved, namely $r_{m-3}^{\prime}=q_{m-3}, q_{m-3}^{\prime}=r_{m-3}$; 
the theorem is, in fact, an extension of the Riemann-Roch theorem on the geometrical side.

If $n$ be less than both $l$ and $m$, and consequently $n^{\prime}$ be greater than both $l-3$ and $m-3$, the first of the formulæ becomes

$$
r_{n^{\prime}}^{\prime}=q_{n}+1
$$

and if $n$ be greater than both $l-3$ and $m-3$, so that $n^{\prime}$ is less than both $l$ and $m$, the second becomes

that is,

$$
\begin{aligned}
& r_{n}=q_{n^{\prime}}{ }^{\prime}+1, \\
& q_{n^{\prime}}{ }^{\prime}=r_{n}-1 .
\end{aligned}
$$

If the difference of $l$ and $m$ is less than 3, one or other of these two cases necessarily presents itself. If the difference of $l$ and $m$ is greater than or equal to 3 , a sufficiently great value of $n(>l-3$ where $l>m)$, or a sufficiently small value $(<m)$, with the accompanying values of $n^{\prime}(<m$ or $>l-3)$, will ensure simplification in one or other of the formulæ.

In the course of the proof of this theorem, Mr. Macaulay establishes the second part of the Cayley theorem, in the form of a criterion for deciding whether the $C_{n}$ that is drawn through $N$ of the intersections of $C_{l}, C_{m}$, passes through the remaining $N^{\prime}(=l m-N)$ points ; the $C_{n}$ does, or does not, pass through these $N^{\prime}$ points according as the $(l+m-n-3)$-ic excess is, or is not, zero. In the use of this criterion a remark made in $\$ 7$ is of importance in determining the excess ; the greatest number of points that can supply independent conditions for curves of order $k$ is $\frac{1}{2}(k+1)(k+2)$, seeing that this number would oblige all the coefficients in $C_{k}$ to vanish. Hence when $N>\frac{1}{2}(k+1)(k+2)$, the $k$-ic excess of $N$ general points is $N-\frac{1}{2}(k+1)(k+2)$.

Having found in any given case that the $N$ points which form the partial intersection of $C_{l}$ and $C_{m}$ have an $n$-ic excess $r_{n}$, is there any way of deciding whether a $C_{n}$ through $N-r_{n}$ of these necessarily passes through the remainder? Mr. Macaulay does not raise this question, but it is irresistibly suggested by the Cayley-Bacharach theorem. The fear of exhibiting a "subtile précipitation de raisonnement" almost dissuades one from attempting to give an answer offhand, though it appears highly probable that this depends solely upon the curve of order $l+m-n-3$ through the $l m-N$ points, which may, or may not, necessarily pass through some of the $N$ points ; those through which it necessarily passes are probably redundant in $N$ as regards the $C_{n}$.

By means of theorem $\mathrm{V}$ we can pass from the character- 
ization of a group $N$ to that of a residual group. On this Mr. Macaulay bases his interesting process, $\$ \S 26-29$, for constructing a point-group of given characterization, for example, a group of 67 points whose excess for curves of order $9,10,11,12$ shall be $13,7,3,1$. The corresponding defects are $0,5,13,24$; hence only one $C_{9}$ can be passed through the points, they form part of the intersection of $C_{9}$ and $C_{10}$, and the residual group contains 23 points. As 12 is the highest order for which the 67 points have an excess, the $n$ of the formula $l+m-n-3$ is 12 , and the lowest order of a curve through the residual 23 is consequently 4. Hence by theorem $\mathrm{V}$,

from which

$$
\begin{aligned}
& q_{4}^{\prime}=r_{12}-1=0, \quad q_{5}{ }^{\prime}=r_{11}-1=2, \\
& q_{6}^{\prime}=r_{10}-1=6, \quad q_{7}{ }^{\prime}=r_{9}-1=12,
\end{aligned}
$$

$$
r_{4}^{\prime}=9, \quad r_{5}^{\prime}=5, \quad r_{6}^{\prime}=2, \quad r_{7}^{\prime}=0 .
$$

That $q_{4}^{\prime}$ is zero agrees with the obvious fact that there is only one $C_{4}$ through the 23 points; and the value 2 for $q_{5}{ }^{\prime}$ shows, what again is otherwise evident, that the only $C_{5}^{\prime}$ 's are those formed by the $C_{4}$ and any straight line. The value 6 for $q_{6}{ }^{\prime}$ shows that there are proper sextics through the points ; hence the residual group $N^{\prime}$ is 23 of the 24 intersections of $C_{4}$ and $C_{6}$; and a group $N$ of the desired characterization is constructed by passing $C_{9}$ and $C_{10}$ through 23 of the intersections of $C_{4}$ and $C_{6}$.

The general process requires that the lowest orders of curves through $N$ be known, $l$ and $m$, where $l \geq m$; if the complete characterization is given, this condition is fulfilled. If $q_{m}>0, l=m$. Let $n$ be the highest order for which the given points have an excess, $n \geq l \geq m$; the $C_{l}$ and $C_{m}$ through $N$ determine a residual $N^{\prime}$; the curve of lowest order through $N^{\prime}$ is $C_{l+m-n-3}$, and curves of all higher orders will pass through $N^{\prime}$. The defects are known from theorem $\mathrm{V}$ in the form

$$
q^{\prime}{ }_{l+m-n-3}=r_{n}-1, \quad q^{\prime}{ }_{l+m-n-2}=r_{n-1}-1, \cdots ;
$$

from these $r^{\prime}{ }_{l+m-n-3}$, etc., are known ; and it is shown that $N^{\prime}$ cannot have an excess for a curve of order greater than $m-3$.

Thus from the characterization of $N$ the characterization of $N^{\prime}$ is known ; and $N^{\prime}$ is less complex than $N$. This is merely stated in the text, but a formal proof, though simple, is hardly superfluous. It is to be shown that $N^{\prime}<N$, $l^{\prime}<l, m^{\prime}<m, n^{\prime}<n$. As $N+N^{\prime}=l m$, it must be shown 
that $N>\frac{1}{2} \mathrm{~lm}$. Now if $N \leq \frac{1}{2} \mathrm{~lm}$, a $C_{l-1}$ could be passed through $N$; for the postulandum of $C_{l-1}$ is $\frac{1}{2}(l-1)(l+2)$, and this is $\geq \frac{1}{2} l m$ if $l^{2}+l-2 \geq l m$, that is, if $l(l-m+1) \geq 2$. As $l \geq m$, and $l \geq 2$, this is satisfied. Thus a $C_{l-1}$ could be passed through $N$, and if $l>m$, more than one such curve, contrary to the hypothesis that $m$ and $l$ are the lowest orders. Hence $N>\frac{1}{2} l m, N^{\prime}<\frac{1}{2} l m$ and therefore $N^{\prime}<N$. Again, it is shown that $n^{\prime} \leq m-3$, hence $n^{\prime}<m$, and therefore $n^{\prime}<n$. Also $l^{\prime}$ and $m^{\prime}$ are both $\leq n^{\prime}$, that is, $<m$, and consequently $<l$. Thus the residual group $N^{\prime}$ contains fewer points than the given one, it is the partial intersection of curves of lower order than any that pass through the given one, and the conditions imposed by it become independent at an earlier stage; it is therefore less complex than the given one. Similarly from $N^{\prime}$ we pass to a simpler group $N^{\prime \prime}$, and so on.

Any obvious impossibility indicates the non-existence of a point-group with the given characterization ; e. g., an attempt to find $*=369$ with excess $48,28,16,7,2$ for curves of order $24,25,26,27,28$, leads to $N^{\prime \prime \prime}=18$, with defects $3,7,17$ for curves of order $5,6,7$. Now 18 points, even if arbitrary, would leave $C_{6}$ with $27-18=9$ degrees of freedom ; hence necessarily $q_{6}^{\prime \prime \prime} \geq 9$, and the calculated value 7 indicates impossibility. Attention would be drawn to this by the resulting negative value, -2 , for $r_{6}^{\prime \prime \prime}$

Of course what is really proved is that the existence of a point-group of the specified type depends on the existence of $N^{\prime}$, this on the existence of $N^{\prime \prime}$, and so on ; thus in every case the last group obtained must be examined with care. This last group, however, will necessarily be of a fairly simple construction; for so long as there are excesses, we can reduce by the given process; and after each step in the reduction the order of the highest curve involved is less by three or more than the order of the lowest curve in the preceding stage.

The process must be applied with due regard to certain conditions, pointed out in $\$ \$ 27,29$; but even with these limitations it is a valuable contribution to the theory of point-groups in a plane. It might with advantage have been brought more prominently forward, with a rather more emphatic statement; as it stands, its importance may be overlooked by a rapid reader. For instance, the reviewer in the Jahrbuch über die Fortschritte der Mathematik, Jahrgang 1895, vol. 26, p. 653, says that the author does not succeed in finding a general law for $r_{n}$; that all that he can show is

* This is a modification of one of Mr. Macaulay's illustrations. 
that the value of $r_{n}$ cannot exceed certain limits. As a matter of fact, the principal thing in Mr. Macaulay's paper is that he does give the law for $r_{n}$. For if the points are entirely arbitrary, $r_{n}$ is in general zero; if they are not arbitrary, something must be given about them; if this be the construction, then these theorems give the values of $r_{n}$, and if the values of $r_{n}$ are given, this process shows the construction.

I have spoken only of the sections that relate to nonsingular curves. The memoir professedly deals also with curves endowed with multiple points of any order, provided all the tangents are distinct; for these multiple points a convention is adopted which strikes mè as unsatisfactory. It would doubtless be convenient if we could describe the nature of a multiple point, with tangents distinct or coincident, in such a way that for all purposes its effect should be the simple sum of the effects of its components. As regards the effect on the class and deficiency, this has been acconplished in ways both geometrical and analytical ; but it has not been done, for example, for the effect on the Jacobian of a net of curves, nor even for the effect on the Hessian of a single curve. Mr. Macaulay's convention of "clusters," $\$ \$ 1,8$, etc., does not seem to me to take us one step further in this line; and his replacement of a curve at a multiple point by a penultimate form, regarding this as an infinitely small curve of definite, though to a certain extent arbitrary, shape-in order to deal with the intersection of two curves at a common multiple point where all the tangents are distinct-is, in my opinion, no advance on the accepted approximation to the curve by means of its tangents.

There are other matters of interest in the memoir, which occupies fifty pages; but they do not come within the scope of this note, in which I have simply tried to give a general account, in what seems to me its proper perspective, of the most striking part of an interesting and valuable paper. Many of the results contained are doubtless well known; but so far as $\mathrm{I}$ am able to judge, there is more that is new than the reviewer in the Jahrbuch is disposed to acknowledge. In this particular line of work the various theorems proceed so directly from their common source that when once recognized a slight twist given to a formula is all that is needed to prove many of them. The real importance of Mr. Macaulay's work, however, consists in the fact that it is a systematic treatment by purely geometrical methods of the interdependence of curves and determining points.

Bryn Mawr College, January, 1898. 